\title{
Beyond Tethering the Viral Particles: Immunomodulatory Functions of Tetherin (BST-2)
}

\author{
Ritudhwaj Tiwari,, Juan C. de la Torre, ${ }^{2}$ Dorian B. McGavern, ${ }^{3}$ and Debasis Nayak ${ }^{1}$
}

Host response to viral infection is a highly regulated process involving engagement of various host factors, cytokines, chemokines, and stimulatory signals that pave the way for an antiviral immune response. The response is manifested in terms of viral sequestration, phagocytosis, and inhibition of genome replication, and, finally, if required, lymphocyte-mediated clearance of virally infected cells. During this process, cross-talk between viral and host factors can shape disease outcomes and immunopathology. Bone marrow stromal antigen 2 (BST-2), also know as tetherin, is induced by type I interferon produced in response to viral infections, as well as in certain cancers. BST-2 has been shown to be a host restriction factor of virus multiplication through its ability to physically tether budding virions and restrict viral spread. However, BST-2 has other roles in the host antiviral response. This review focuses on the diverse functions of $B S T-2$ and its downstream signaling pathways in regulating host immune responses.

Keywords: tetherin, immunomodulatory, antiviral, cancer

\section{History and Molecular Characterization}

I NITIALLY DISCOVERED AS A SURFACE MARKER for terminally differentiated and neoplastic B cells, bone marrow stromal antigen-2 (BST-2) was later reported to have diverse cellular functions (Goto et al., 1994; Ishikawa et al., 1995). $B S T-2$ is widely expressed but its levels vary from cell to cell (Erikson et al., 2011; Hanagata and Li, 2011; Jones et al., 2012). BST-2 is a type II transmembrane protein and contains $\sim 180$ amino acids (aas). The mature protein adopts a unique topology comprising a short $N$-terminal cytoplasmic tail (1-20 aas) followed by an $\alpha$-helical transmembrane domain (21-48 aas), an ectodomain (49-161 aas), and a C-terminal glycosylphosphatidylinositol (GPI) domain (162-180 aas) (Kupzig et al., 2003; Hinz et al., 2010).

The cytoplasmic tail of BST-2 has a highly conserved YXY tyrosine motif known to play a role in $\mathrm{NF}-\kappa \mathrm{B}-$ mediated signaling and clathrin-mediated endocytosis (Masuyama et al., 2009; Tokarev et al., 2009; Galao et al., 2012), whereas its ectodomain contains three cysteine residues and two glycosylation sites that are highly conserved during evolution (Andrew et al., 2009). These conserved cysteine residues covalently link monomers to form dimeric or tetrameric forms of BST-2 (Swiecki et al., 2011). Two $N$-linked glycosylation sites (at N65 and N92) are required for the proper folding of $B S T-2$, and the GPI domain anchors
$B S T-2$ to cell surface lipid rafts. BST-2 expression is present at the plasma membrane, as well as in the transGolgi network and within recycling endosomes (Hammonds et al., 2010).

\section{Antiviral Activity of BST-2}

In 2008, Bieniasz and Guatelli groups independently reported that $B S T$-2 restricted the release of human immunodeficiency virus-1 (HIV-1). BST-2 inhibited virion release of a recombinant HIV-1 lacking the $V p u$ gene by tethering the nascent virion to the host cell plasma membrane (Neil et al., 2008; Van Damme et al., 2008). This prompted the name "tetherin" for BST-2. Subsequently, BST-2 was shown to tether a broad range of enveloped viruses, but its tethering activity against enveloped viruses is not a universal phenomenon. The antiviral activity of $B S T-2$ is related to its membrane anchoring topology (Andrew et al., 2009). The two anchoring domains of BST-2 form a bridge between the budding virion and the host plasma membrane, thereby physically restricting virion release (Neil et al., 2008; Van Damme et al., 2008). Perez Caballero et al. (2009) demonstrated that the membrane anchoring domains are necessary and sufficient for viral tethering. The antiviral activity of tetherin is independent of other host factors. BST-2 transmembrane domain, dimeric ectodomain,

\footnotetext{
${ }^{1}$ Discipline of Bioscience and Biomedical Engineering, Indian Institute of Technology Indore, Indore, India.

${ }^{2}$ Department of Immunology and Microbial Science IMM-6, The Scripps Research Institute, La Jolla, California.

${ }^{3}$ Viral Immunology \& Intravital Imaging Section, National Institute of Neurological Disorders and Stroke, National Institutes of Health, Bethesda, Maryland.
} 
and GPI anchor domains are critical for BST-2 antiviral activities. Tethered virions are either retained at the cell surface or mobilized for endocytic internalization and subsequent ubiquitin-based degradation (Neil et al., 2006; Miyakawa et al., 2009). BST-2 antiviral activities against a variety of enveloped viruses, including retroviruses, alphaviruses, rhabdoviruses, and mammarenaviruses, have been recently discussed in several excellent review articles (Tokarev et al., 2009; Evans et al., 2010; Arias et al., 2011; Sarojini et al., 2011; Swiecki et al., 2013; Mahauad-Fernandez and Okeoma, 2016). This review highlights other aspects of $B S T-2$ biology, including cell signaling, immunomodulatory functions, and immunity.

\section{BST-2 and Cell Signaling Pathways}

$B S T-2$ expression is induced by type I and type II interferons (IFNs) in response to viral infection. The role of $B S T-2$ in cell biology was first illustrated by its potent induction of $N F-\kappa B$ (Matsuda et al., 2003). More recent studies confirmed a role for $B S T-2$ in regulating $N F-\kappa B$ signaling (Cocka and Bates, 2012; Galao et al., 2012; Tokarev et al., 2013). BST-2 induction of $N F-\kappa B$ is dependent upon its multimerization or viral sensing actions (Tokarev et al., 2013). Earlier studies identified TGF beta-activated kinase 1 (TAK1) as being critical for $B S T$-2-induced $N F-\kappa B-$ mediated signal transduction (Galao et al., 2012; Tokarev et al., 2013). However, subsequent studies identified additional intermediate signaling molecules with roles in activation of the $N F-\kappa B$ signaling pathway by $B S T-2$. Thus, knockdown of TNF receptor associated factor 6 (TRAF6) or TNF receptor associated factor 2 (TRAF2)/ubiquitinconjugating enzyme E2N (Ubc13) gene expression blocked $B S T-2-$ mediated $N F-\kappa B$ activation (Galao et al., 2012). In contrast, myeloid differentiation primary response protein MyD88, TRAF2, TAK1, TAK1-binding protein 1 (TAB1), and TAK1-binding protein 2 (TAB2) were found dispensable for $B S T$-2-induced activation of the $N F-\kappa B$ signaling pathway (Tokarev et al., 2013). These findings revealed that $B S T-2$, through induction of $N F-\kappa B$ signaling, can influence the host inflammatory response to a virus (Moynagh, 2005; Liu et al., 2017b), but the underlying mechanisms remain to be elucidated.

\section{Immunomodulatory Role of BST-2 After Infection}

\section{IFNs and innate immunity}

Upon pathogen encounter, pattern recognition receptors including Toll-like receptors (TLRs), Nod-like receptors (NLRs), and RIG-1-like receptors (RLRs) (Goubau et al., 2013; Cao, 2015; Chan and Gack, 2016; Liu et al., 2017a) can initiate antiviral responses, including IFN-I and proinflammatory cytokine production (Takeuchi and Akira, 2010). IFN-I signaling through the IFN receptor and JAK/STAT pathway induces expression of hundreds of IFN-stimulated genes that contribute to the establishment of an antiviral state that limits virus propagation within the infected host (Schneider et al., 2014). IFN-I responses are highly controlled and short lived, but if unchecked, excessive expression of IFNs may harm the host (Gota and Calabrese, 2003) and negatively affect hematopoiesis (Lin et al., 1998).
Plasmacytoid dendritic cells (pDCs) are among the highest producers of IFNs and inflammatory cytokines upon sensing bacterial or viral nucleic acids through TLR7 and TLR9 receptors (Colonna et al., 2004; Honda and Taniguchi, 2006). $B S T-2$ negatively regulates the IFN-I response in pDCs (Cao et al., 2009). BST-2 is a biological ligand for the human $\mathrm{pDC}-$ specific receptor immunoglobin-like transcript 7 (ILT7), and binding of $B S T-2$ to $I L T 7$ can initiate signaling through the ILT7-FceRIy (a high-affinity IgE receptor) complex. FceRIy contains an immunoreceptor tyrosine-based activation motif in its cytoplasmic tail that mediates a calcium-dependent signaling cascade that inhibits the production of IFNs and inflammatory cytokines by pDCs (Cho et al., 2008; Cao et al., 2009). However, the detailed mechanisms underlying this negative feedback loop are largely unknown. Coculture of pDCs with $B S T$-2-expressing cells reduced IFN-I production by pDCs after stimulation by the oligodeoxynucleotide, CpGA (Janovec et al., 2018). Moreover, treatment with a MEK1/ 2 inhibitor significantly increased IFN production (Janovec et al., 2018), suggesting that the BST-2-ILT7-mediated downregulation of IFN-I is at least partially associated with MEK1/2 signaling. This pathway is highly specific for human pDCs, as ILT7 is only present in human and primate pDCs (Brown et al., 2004).

$B S T$-2-mediated downregulation of the IFN-I response was also linked to the ability of this protein to counteract the RLR-mediated IFN-I signaling pathway (Jin et al., 2017). Specifically, BST-2 recruits the E3 ubiquitin ligase, MARCH 8, which catalyzes the lysine (K27) linked polyubiquitin chains on the mitochondrial antiviral-signaling protein (MAVS) (Jin et al., 2017), which targets MAVS for autophagic degradation through nuclear domain 10 protein 52 (NDP52) receptor. As MAVS is an essential host signaling adaptor protein for IFN-I production, its degradation negatively affected the IFN response (Jin et al., 2017) (Fig. 1). Interestingly, murine NDP52 lacks the ubiquitinbinding domain LIM-L (Thurston et al., 2009; Inomata et al., 2012; Deretic et al., 2013), which should prevent $B S T-2-$ mediated degradation of MAVS. In fact, pDCs from $B S T-2$ knockout mice showed reduced IFN-I secretion in response to viral challenges (Swiecki et al., 2012). These results suggest that evolutionary selection of the LIM-L domain in NDP-52 and ILT7 expression by human pDCs cells influences how $B S T-2$ regulates IFN-I production in these cells relative to mouse pDCs. Additional research is required to determine how $B S T-2$ affects the innate antiviral immune response in different species.

\section{Adaptive immunity}

Although BST-2 has conserved coding regions, polymorphic forms do exist across species. Thus, NZW/LacJ (NZW) mice contain BST-2 alleles lacking the endosomalsorting motif $(\mathrm{YxY})$ and show higher $B S T$-2 cell surface expression than C57BL/6 mice, whose BST-2 contains the YxY motif. Consistent with its higher cell surface expression, $B S T$-2 from NZW mice exhibited a more potent antiviral activity against Friend murine leukemia retrovirus (F-MuLV) than BST-2 from C57BL/6 mice (Barrett et al., 2012). However, the endocytosis-competent version of $B S T-2$ in C57BL/6 mice showed a greater ability to control viremia, suggesting a role for immune modulatory functions 


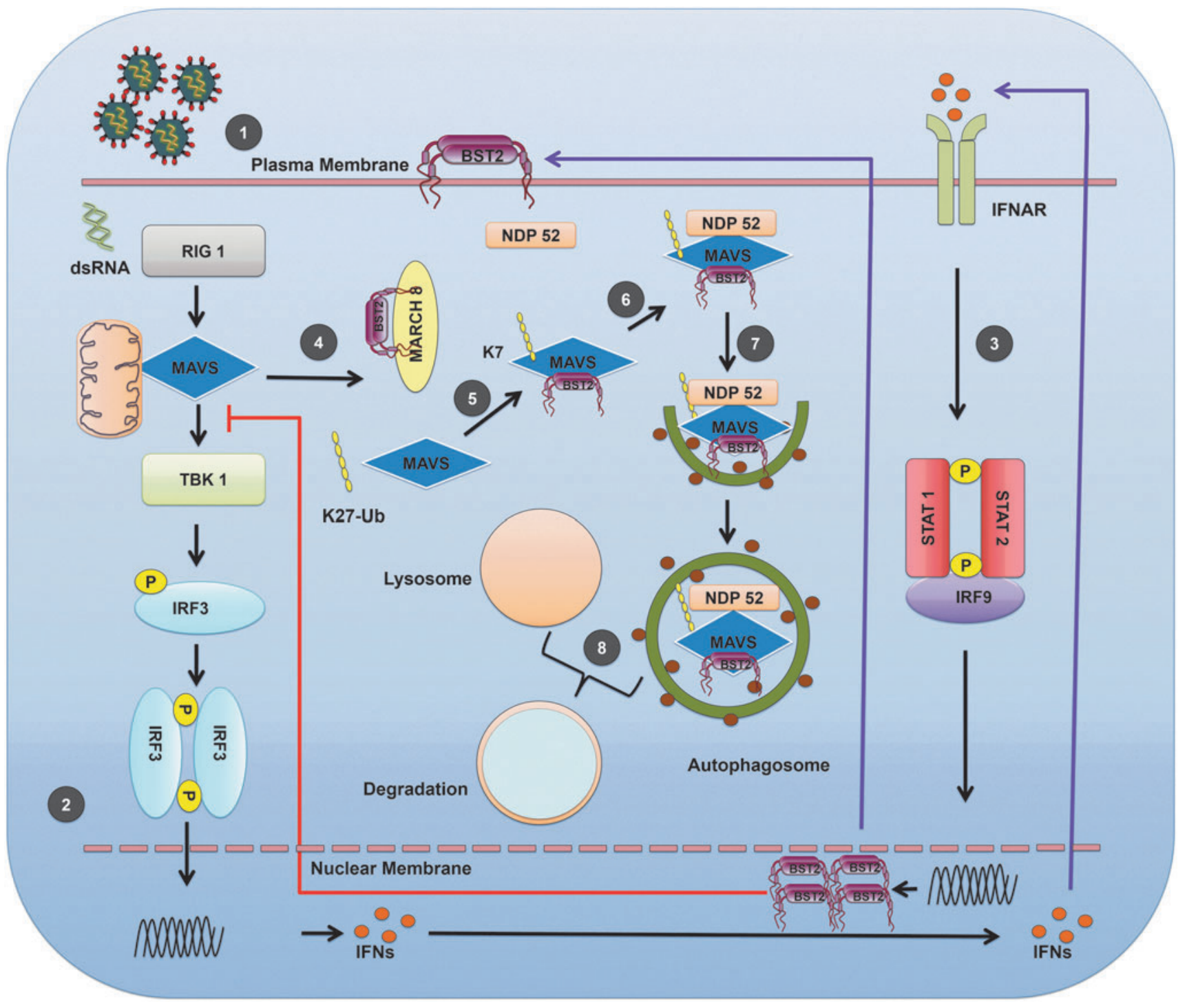

FIG. 1. BST-2 regulation of the IFN-I response. (1) Virion interacts with cell surface receptors to enter the cell. (2) Viral genome (RNA) is recognized by the RLRs. RIG-I signals are transduced to the transcription factors through stimulation of $M A V S$ at the mitochondrion-associated membrane. Activation of MAVS leads to phosphorylation of IRF3. Phosphorylated dimers of IRF3 then translocate to the nucleus where they bind and activate specific promoters triggering expression of IFNs. (3) Type-I IFNs interact with IFNAR, recruit, and phosphorylate the STAT1 and STAT2. STAT1 and STAT2 form a heterodimer that, in turn, recruits the IRF9 to make a complex. This complex translocates to the nucleus and induces expression of genes (e.g., BST-2) regulated by IFN-stimulated response elements. (4) BST-2 recruits the E3 ubiquitin ligase $M A R C H$ 8. (5) $M A R C H$ 8 then catalyzes the K27-linked polyubiquitin chains on MAVS at K7 position. (6) Cargo receptor NDP52 recognizes ubiquitinated MAVS. (7) NDP52 delivers MAVS to autophagosome for degradation. (8) BST-2-mediated autosomal degradation of $M A V S$ and terminal of RIG-I, MAVS-mediated IFNI production via a negative feedback manner. BST-2, bone marrow stromal antigen 2; IFN, interferon; IRF3, IFN response factor 3; IFNAR, IFN- $\alpha / \beta$ receptor; $M A V S$, mitochondrial antiviral-signaling protein; RLR, RIG-1-like receptor; STAT, signal transducers and activators of transcription.

linked to BST-2 (Li et al., 2014). Consistent with this hypothesis, enhanced restriction of F-MuLV in C57BL/6 mice was associated with a stronger IFN $\gamma$ response in NK cells, $\mathrm{CD}^{+} \mathrm{T}$ cells, and $\mathrm{CD}^{+} \mathrm{T}$ cells (Li et al., 2014). Increased endocytosis of virions by $\mathrm{pDCs}$ in $\mathrm{C} 57 \mathrm{BL} / 6$ mice could trigger TLR3-mediated IFN-I production, leading to augmented NK function, as these cells are highly responsive to TLR3- and TLR7-dependent cytokine stimuli (Swiecki et al., 2012; Gibbert et al., 2014; Li et al., 2014, 2016) (Fig. 2A). This might account for lower IFN-I production observed in BST-2-deficient pDCs (Swiecki et al., 2012). Because BST-2 can influence IFN-I levels, it has the potential to modulate host defense during both the early and late phases of viral infection. Accordingly, using the mouse model of chronic infection by lymphocytic choriomeningitis virus (LCMV), we showed that the early confinement and sequestration of virions in the splenic marginal zone were compromised in the absence of BST-2 (Urata and Kenyon, 2018). This resulted in alterations in antiviral T cell priming, leading to reduced $\mathrm{T}$ cell proliferation and effector functions 


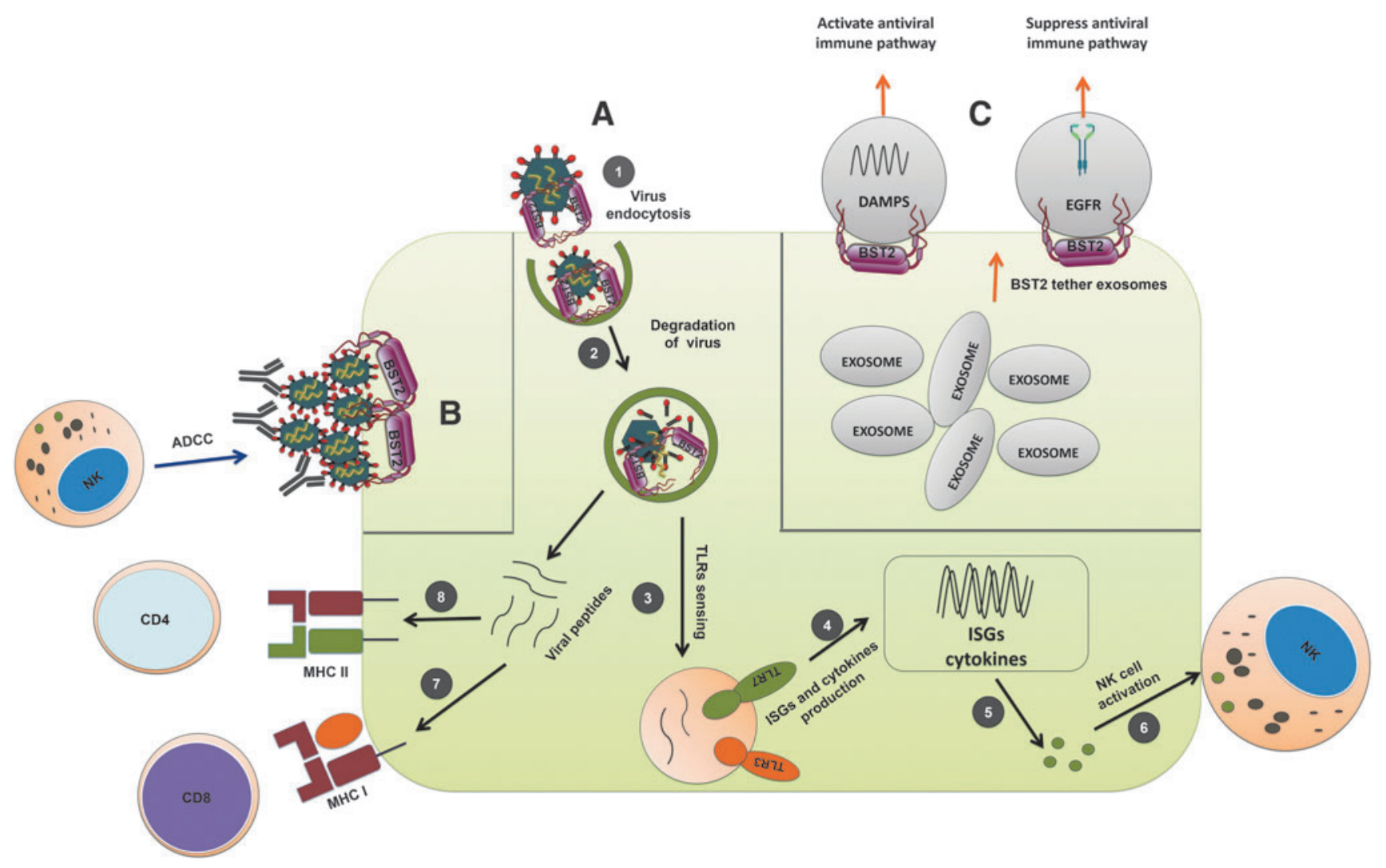

FIG. 2. Antiviral and immunomodulatory functions of BST-2. (A) (1) BST-2 interacts with viral envelope and restricts cellular egress of nascent virion that, in turn, internalizes the virion through endocytosis. (2) In addition, endosomally expressed BST-2 halts virion trafficking and likely allows more time for endosomal proteases to act upon and degrade the virions. (3) Endosomal degradation of viral envelope facilitates release of genomic RNA that activates TLR3 and TLR7mediated innate immune pathways. $(4,5)$ Activation of TLR3 and TLR7 along with other costimulatory molecules can further enhance expression of ISGs and cytokines in antigen presenting cells. (6) Cytokines such as $I L-15$ can promote NK cell activation and function. (7) Proteolytically degraded viral proteins generate a plethora of viral peptides that are often cross-presented by MHC I and can stimulate CD8 T cells. (8) Similarly, peptides loaded into MHC II can promote CD4 T cell activation. (B) In HIV-I-infected cells, the interaction between BST-2 and viral Env protein can increase accumulation of Env at the surface of the cell. This can facilitate interactions with circulating antibodies against HIV-1 and stimulate ADCC-mediated elimination of the infected cell. (C) BST-2 can tether exosomes like viral envelopes restricting their movement. Exosomes often carry signaling molecules such as DAMPs and activated EGFR. DAMP-carrying exosomes can activate antiviral immunity, whereas EGFR-carrying exosomes can suppress it. ADCC, antibody-dependent cellular cytotoxicity; DAMPs, damage-associated molecular patterns; EGFR, epidermal growth factor receptor; HIV-1, human immunodeficiency virus-1; IL, interleukin; ISGs, IFN-stimulated genes; TLR, Toll-like receptor.

(e.g., IFN $\gamma$ and $T N F \alpha$ ). Peripheral control of a chronic LCMV infection was also compromised in BST-2-deficient mice, and the virus established long-term persistence in the brain (Urata and Kenyon, 2018). These findings illustrated how BST-2 can influence both innate and adaptive immune responses to viral infections. Further studies are required to understand the direct versus indirect contributions of BST-2 to antiviral immunity, and how the functionality of this protein can be enhanced to help fight infections.

The relationship between $B S T-2$ and antibody-dependent cellular cytotoxicity (ADCC) in HIV-1-infected cells is well established (Arias et al., 2014). Higher surface expression of Env in infected cells could facilitate ADCC. HIV-1 mutants with impaired Vpu-mediated BST-2 counteracting activity show increased surface accumulation of Env protein in infected cells, which can facilitate ADCC. Thus BST-2 increases the susceptibility of $V p u$-mutated HIV-infected cells to ADCC and knockdown of BST-2 decreases the sensitivity of HIV-infected cells to ADCC (Arias et al., 2014). Notably, overexpression of $B S T$-2 in response to type I IFN, interleukin (IL)-27 or by other means, upregulates surface expression of Env protein and further stimulates HIV-1-infected cells for ADCC-mediated elimination (Pham et al., 2016; Richard et al., 2017) (Fig. 2B). These studies demonstrate an indirect connection of BST-2 and ADCC phenomena in HIV-1-infected cells, although this phenomenon is not yet reported in other viral infection models. Therefore, BST-2 could modulate interplay between innate and adaptive immune response to control viral replication.

\section{Other immunomodulation roles of BST-2}

Various other immunomodulation functions of BST-2 have also been reported. For example, in addition to tethering of 
viral envelope, it also reported to tether exosomes and could influence its cellular target and interactions (Edgar et al., 2016). Exosomes are involved in host immune responses and linked with various diseases such as cancer and neurodisorders (Soria et al., 2017; Tai et al., 2018). In case of cancer, exosome carries damage-associated molecular patterns (DAMPs) in myeloid cells that stimulate inflammatory cytokine productions and could promote cancer progression (Hoshino et al., 2015; Nabet et al., 2017). Alternatively, tumor exosomes could also suppress innate antiviral immune response by transferring the activated epidermal growth factor receptor (EGFR) to the dendritic cells (Gao et al., 2018) (Fig. 2C). Thus BST-2's tethering action on exosome could influence outcome of host immune response to tumor. In a different model, Sally James et al. identified a new subset of nondifferentiating bone marrow stromal cells (BMSCs), which exclusively expresses higher $B S T-2$ and IL-7, although specific functional roles of $B S T-2$ in these BMSCs are yet to be defined ( James et al., 2015). A similar observation was made wherein higher cells surface expression of BST-2 was noticed in cancellous bone fragments resident cells (El-Sherbiny et al.,
2018). However, relevance of BST-2 expression and its mechanistic role is yet to be elucidated.

\section{BST-2 and Cancer}

Increased BST-2 expression has been documented in different cancer tissues, including ovarian, lung, head and neck, cervical, thyroid, breast, endometrial, pancreatic glioblastoma, and myeloma (Wang et al., 2009; Wainwright et al., 2011; Tai et al., 2012; Yokoyama et al., 2013; Fang et al., 2014; Mahauad-Fernandez et al., 2014, 2015; Milutin Gasperov et al., 2014). BST-2 overexpression at the early stage of multiple myeloma suggested that BST-2 might be a suitable target for cancer immune therapy. However, not all cancer types exhibit increased BST-2 expression. BST-2 expression is unchanged in thyroid and lung adenocarcinomas and is downregulated in liver, kidney, lung squamous, and prostate cancer relative to normal cells (Mahauad-Fernandez et al., 2015). Almost all breast tumors express BST-2 to a certain level, and a higher expression level of $B S T-2$ is associated with aggressive and progressive malignancy

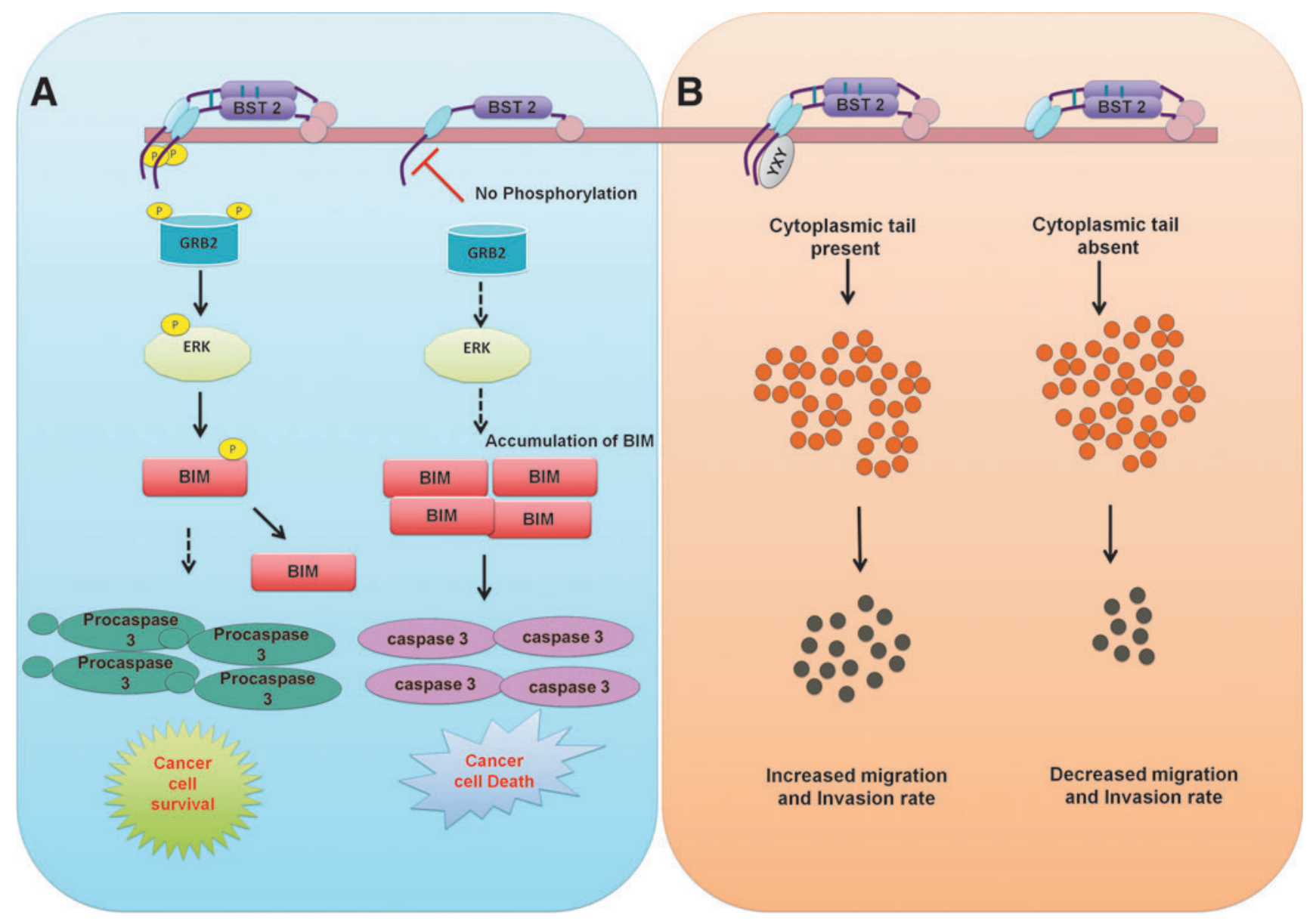

FIG. 3. The influence of $B S T-2$ on tumor cell survival, invasion, and migration. (A) The dimeric form of $B S T-2$ can facilitate cell-to-cell interactions or extracellular matrix interactions. BST-2 activation leads to phosphorylation of its cytoplasmic tail (most likely in the tyrosine-6 and tyrosine-8 positions). Phosphorylated $B S T-2$ recruits $G R B-2$ and activates a kinase (unknown) that phosphorylates $E R K$ (pERK), which, in turn, phosphorylates BIM, resulting in subsequent proteasomal degradation of $B I M$. In the absence of $B I M$, procaspase-3 is neither cleaved nor activated. This results in cancer cell survival. In a monomeric form, cytoplasmic domain of $B S T-2$ is not phosphorylated, which can promote apoptosis of cancer cells. (B) The YXY motif of BST-2 is responsible for cancer cell migration and invasion. In the absence of the YXY motif, cancer cells exhibit a reduced migration rate. 
(Mahauad-Fernandez et al., 2014).The functional significance of $B S T-2$ expression levels in malignancy remains to be elucidated, but $B S T-2$ homodimers appear critical in certain instances for the promotion of cancer cell adhesion (Mahauad-Fernandez and Okeoma, 2017). In addition, BST2 enhances cancer cell survival and growth by promoting proteosomal degradation of proapoptotic proteins, such as BIM - a member of the Bcl-2 protein family (MahauadFernandez and Okeoma, 2017) (Fig. 3A). Evidence indicates that the cytoplasmic tail of BST-2 is responsible for cell migration and invasion, but the mechanisms underlying such functions are largely unknown (Naushad et al., 2017) (Fig. 3B). Studying the role of BST-2 in different cancers is a very active area of research, and in future we expect that novel insights will emerge regarding the relationship between $B S T-2$ and malignancy.

\section{Conclusion}

Cells have an array of factors that play precise roles in maintaining normal physiology and responding to diverse challenges (infections, tumors, etc.). A plethora of factors have been discovered that contribute to host defense against pathogens and the maintenance of immune homeostasis. $B S T-2$ is one such factor, and studies have highlighted multifaceted biological roles for $B S T-2$ in antiviral responses, cell signaling, immune modulation, and even malignancy. Because the immune system has the potential to cause pathology, it is important that responses are precisely regulated. A role for $B S T-2$ in immune modulation is supported by its ability to alter IFN-I, $N F-\kappa B$, and T cell effector functions. The detailed mechanisms by which $B S T-2$ executes these functions remain to be elucidated, but future studies are likely to uncover a broader role for $B S T$-2 in shaping host responses to infection and cancer. This knowledge may facilitate the development of therapeutics based on regulating $B S T-2$ functionality.

\section{Authors' Contributions}

R.T. and D.N. structured the article and gave their inputs toward shaping the article. J.C.d.I.T. and D.B.M. made critical suggestions and editing the article.

\section{Disclosure Statement}

No competing financial interest exists.

\section{Funding Information}

R.T. is thankful to University Grant Commission (UGC), Govt. of India, for his graduate fellowship.

\section{References}

Andrew, A.J., Miyagi, E., Kao, S., and Strebel, K. (2009). The formation of cysteine-linked dimers of BST-2/tetherin is important for inhibition of HIV-1 virus release but not for sensitivity to Vpu. Retrovirology $\mathbf{6}, 80$.

Arias, J.F., Heyer, L.N., von Bredow, B., Weisgrau, K.L., Moldt, B., Burton, D.R., et al. (2014). Tetherin antagonism by $\mathrm{Vpu}$ protects HIV-infected cells from antibody-dependent cell-mediated cytotoxicity. Proc Natl Acad Sci U S A 111, 6425-6430.
Arias, J.F., Iwabu, Y., and Tokunaga, K. (2011). Structural basis for the antiviral activity of BST-2/tetherin and its viral antagonism. Front Microbiol 2, 250.

Barrett, B.S., Smith, D.S., Li, S.X., Guo, K., Hasenkrug, K.J., and Santiago, M.L. (2012). A single nucleotide polymorphism in tetherin promotes retrovirus restriction in vivo. PLoS Pathog 8, e1002596.

Brown, D., Trowsdale, J., and Allen, R. (2004). The LILR family: modulators of innate and adaptive immune pathways in health and disease. Tissue Antigens 64, 215-225.

Cao, W., Bover, L., Cho, M., Wen, X., Hanabuchi, S., Bao, M., et al. (2009). Regulation of TLR7/9 responses in plasmacytoid dendritic cells by BST2 and ILT7 receptor interaction. J Exp Med 206, 1603-1614.

Cao, X. (2015). Self-regulation and cross-regulation of patternrecognition receptor signalling in health and disease. Nat Rev Immunol 16, 35 .

Chan, Y.K., and Gack, M.U. (2016). Viral evasion of intracellular DNA and RNA sensing. Nat Rev Microbiol 14, 360-373.

Cho, M., Ishida, K., Chen, J., Ohkawa, J., Chen, W., Namiki, S., et al. (2008). SAGE library screening reveals ILT7 as a specific plasmacytoid dendritic cell marker that regulates type I IFN production. Int Immunol 20, 155-164.

Cocka, L.J., and Bates, P. (2012). Identification of alternatively translated Tetherin isoforms with differing antiviral and signaling activities. PLoS Pathog 8, e1002931.

Colonna, M., Trinchieri, G., and Liu, Y.J. (2004). Plasmacytoid dendritic cells in immunity. Nat Immunol 5, 1219-1226.

Deretic, V., Saitoh, T., and Akira, S. (2013). Autophagy in infection, inflammation and immunity. Nat Rev Immunol 13, 722-737.

Edgar, J.R., Manna, P.T., Nishimura, S., Banting, G., and Robinson, M.S. (2016). Tetherin is an exosomal tether. Elife 5, pii: e17180.

El-Sherbiny, Y.M., El-Jawhari, J.J., Moseley, T.A., McGonagle, D., and Jones, E. (2018). T cell immunomodulation by clinically used allogeneic human cancellous bone fragments: a potential novel immunotherapy tool. Sci Rep 8, 13535.

Erikson, E., Adam, T., Schmidt, S., Lehmann-Koch, J., Over, B., Goffinet, C., et al. (2011). In vivo expression profile of the antiviral restriction factor and tumor-targeting antigen CD317/BST-2/HM1.24/tetherin in humans. Proc Natl Acad Sci U S A 108, 13688-13693.

Evans, D.T., Serra-Moreno, R., Singh, R.K., and Guatelli, J.C. (2010). BST-2/tetherin: a new component of the innate immune response to enveloped viruses. Trends Microbiol 18, 388-396.

Fang, K.H., Kao, H.K., Chi, L.M., Liang, Y., Liu, S.C., Hseuh, C., et al. (2014). Overexpression of BST2 is associated with nodal metastasis and poorer prognosis in oral cavity cancer. Laryngoscope 124, E354-E360.

Galao, R.P., Le Tortorec, A., Pickering, S., Kueck, T., and Neil, S.J. (2012). Innate sensing of HIV-1 assembly by Tetherin induces NFkappaB-dependent proinflammatory responses. Cell Host Microbe 12, 633-644.

Gao, L., Wang, L., Dai, T., Jin, K., Zhang, Z., Wang, S., et al. (2018). Tumor-derived exosomes antagonize innate antiviral immunity. Nat Immunol 19, 233-245.

Gibbert, K., Francois, S., Sigmund, A.M., Harper, M.S., Barrett, B.S., Kirchning, C.J., et al. (2014). Friend retrovirus drives cytotoxic effectors through Toll-like receptor 3. Retrovirology 11, 126.

Gota, C., and Calabrese, L. (2003). Induction of clinical autoimmune disease by therapeutic interferon- $\alpha$. Autoimmunity 36, 511-518. 
Goto, T., Kennel, S.J., Abe, M., Takishita, M., Kosaka, M., Solomon, A., et al. (1994). A novel membrane antigen selectively expressed on terminally differentiated human B cells. Blood 84, 1922-1930.

Goubau, D., Deddouche, S., and Reis e Sousa, C. (2013). Cytosolic sensing of viruses. Immunity 38, 855-869.

Hammonds, J., Wang, J.J., Yi, H., and Spearman, P. (2010). Immunoelectron microscopic evidence for Tetherin/BST2 as the physical bridge between HIV-1 virions and the plasma membrane. PLoS Pathog 6, e1000749.

Hanagata, N., and Li, X. (2011). Osteoblast-enriched membrane protein IFITM5 regulates the association of CD9 with an FKBP11-CD81-FPRP complex and stimulates expression of interferon-induced genes. Biochem Biophys Res Commun 409, 378-384.

Hinz, A., Miguet, N., Natrajan, G., Usami, Y., Yamanaka, H., Renesto, P., et al. (2010). Structural basis of HIV-1 tethering to membranes by the BST-2/tetherin ectodomain. Cell Host Microbe 7, 314-323.

Honda, K., and Taniguchi, T. (2006). IRFs: master regulators of signalling by Toll-like receptors and cytosolic patternrecognition receptors. Nat Rev Immunol 6, 644-658.

Hoshino, A., Costa-Silva, B., Shen, T.L., Rodrigues, G., Hashimoto, A., Tesic Mark, M., et al. (2015). Tumour exosome integrins determine organotropic metastasis. Nature 527, 329-335.

Inomata, M., Niida, S., Shibata, K., and Into, T. (2012). Regulation of Toll-like receptor signaling by NDP52-mediated selective autophagy is normally inactivated by A20. Cell Mol Life Sci 69, 963-979.

Ishikawa, J., Kaisho, T., Tomizawa, H., Lee, B.O., Kobune, Y., Inazawa, J., et al. (1995). Molecular cloning and chromosomal mapping of a bone marrow stromal cell surface gene, BST2, that may be involved in pre-B-cell growth. Genomics 26, 527-534.

James, S., Fox, J., Afsari, F., Lee, J., Clough, S., Knight, C., et al. (2015). Multiparameter analysis of human bone marrow stromal cells identifies distinct immunomodulatory and differentiation-competent subtypes. Stem Cell Reports 4, 1004-1015.

Janovec, V., Aouar, B., Font-Haro, A., Hofman, T., Trejbalova, K., Weber, J., et al. (2018). The MEK1/2-ERK pathway inhibits type I IFN production in plasmacytoid dendritic cells. Front Immunol 9, 364.

Jin, S., Tian, S., Luo, M., Xie, W., Liu, T., Duan, T., et al. (2017). Tetherin suppresses type I interferon signaling by targeting MAVS for NDP52-mediated selective autophagic degradation in human cells. Mol Cell 68, 308-322.e4.

Jones, P.H., Mehta, H.V., Maric, M., Roller, R.J., and Okeoma, C.M. (2012). Bone marrow stromal cell antigen 2 (BST-2) restricts mouse mammary tumor virus (MMTV) replication in vivo. Retrovirology 9, 10.

Kupzig, S., Korolchuk, V., Rollason, R., Sugden, A., Wilde, A., and Banting, G. (2003). Bst-2/HM1.24 is a raft-associated apical membrane protein with an unusual topology. Traffic 4, 694-709.

Li, S.X., Barrett, B.S., Guo, K., and Santiago, M.L. (2016). Tetherin/BST-2: restriction factor or immunomodulator? Curr HIV Res 14, 235-246.

Li, S.X., Barrett, B.S., Heilman, K.J., Messer, R.J., Liberatore, R.A., Bieniasz, P.D., et al. (2014). Tetherin promotes the innate and adaptive cell-mediated immune response against retrovirus infection in vivo. $\mathrm{J}$ Immunol 193, 306-316.
Lin, Q., Dong, C., and Cooper, M.D. (1998). Impairment of $\mathrm{T}$ and $\mathrm{B}$ cell development by treatment with a type I interferon. J Exp Med 187, 79-87.

Liu, B., Zhang, M., Chu, H., Zhang, H., Wu, H., Song, G., et al. (2017a). The ubiquitin E3 ligase TRIM31 promotes aggregation and activation of the signaling adaptor MAVS through Lys63-linked polyubiquitination. Nat Immunol 18, 214-224.

Liu, T., Zhang, L., Joo, D., and Sun, S.-C. (2017b). NF-кB signaling in inflammation. Signal Transduction And Targeted Therapy 2, 17023.

Mahauad-Fernandez, W.D., Borcherding, N.C., Zhang, W., and Okeoma, C.M. (2015). Bone marrow stromal antigen 2 (BST-

2) DNA is demethylated in breast tumors and breast cancer cells. PLoS One 10, e0123931.

Mahauad-Fernandez, W.D., DeMali, K.A., Olivier, A.K., and Okeoma, C.M. (2014). Bone marrow stromal antigen 2 expressed in cancer cells promotes mammary tumor growth and metastasis. Breast Cancer Res 16, 493.

Mahauad-Fernandez, W.D., and Okeoma, C.M. (2016). The role of BST-2/Tetherin in host protection and disease manifestation. Immun Inflamm Dis 4, 4-23.

Mahauad-Fernandez, W.D., and Okeoma, C.M. (2017). Cysteine-linked dimerization of BST-2 confers anoikis resistance to breast cancer cells by negating proapoptotic activities to promote tumor cell survival and growth. Cell Death Dis 8, e2687.

Masuyama, N., Kuronita, T., Tanaka, R., Muto, T., Hirota, Y., Takigawa, A., et al. (2009). HM1.24 is internalized from lipid rafts by clathrin-mediated endocytosis through interaction with alpha-adaptin. J Biol Chem 284, 15927-15941.

Matsuda, A., Suzuki, Y., Honda, G., Muramatsu, S., Matsuzaki, O., Nagano, Y., et al. (2003). Large-scale identification and characterization of human genes that activate NF-kappaB and MAPK signaling pathways. Oncogene 22, 3307-3318.

Milutin Gasperov, N., Farkas, S.A., Nilsson, T.K., and Grce, M. (2014). Epigenetic activation of immune genes in cervical cancer. Immunol Lett 162, 256-257.

Miyakawa, K., Ryo, A., Murakami, T., Ohba, K., Yamaoka, S., Fukuda, M., et al. (2009). BCA2/Rabring7 promotes tetherindependent HIV-1 restriction. PLoS Pathog 5, e1000700.

Moynagh, P.N. (2005). The NF-kappaB pathway. J Cell Sci 118, 4589-4592.

Nabet, B.Y., Qiu, Y., Shabason, J.E., Wu, T.J., Yoon, T., Kim, B.C., et al. (2017). Exosome RNA unshielding couples stromal activation to pattern recognition receptor signaling in cancer. Cell 170, 352-366 e13.

Naushad, W., Mahauad-Fernandez, W.D., and Okeoma, C.M. (2017). Structural determinant of BST-2-mediated regulation of breast cancer cell motility: a role for cytoplasmic tail tyrosine residues. Oncotarget 8, 110221-110233.

Neil, S.J., Eastman, S.W., Jouvenet, N., and Bieniasz, P.D. (2006). HIV-1 Vpu promotes release and prevents endocytosis of nascent retrovirus particles from the plasma membrane. PLoS Pathog 2, e39.

Neil, S.J., Zang, T., and Bieniasz, P.D. (2008). Tetherin inhibits retrovirus release and is antagonized by HIV-1 Vpu. Nature 451, 425-430.

Perez-Caballero, D., Zang, T., Ebrahimi, A., McNatt, M.W., Gregory, D.A., Johnson, M.C., et al. (2009). Tetherin inhibits HIV-1 release by directly tethering virions to cells. Cell 139, 499-511.

Pham, T.N., Lukhele, S., Dallaire, F., Perron, G., and Cohen, E.A. (2016). Enhancing virion tethering by BST2 sensitizes productively and latently HIV-infected $\mathrm{T}$ cells to ADCC 
mediated by broadly neutralizing antibodies. Sci Rep 6, 37225.

Richard, J., Prevost, J., von Bredow, B., Ding, S., Brassard, N., Medjahed, H., et al. (2017). BST-2 expression modulates small CD4-mimetic sensitization of HIV-1-infected cells to antibody-dependent cellular cytotoxicity. J Virol 91, pii: e00219-17.

Sarojini, S., Theofanis, T., and Reiss, C.S. (2011). Interferoninduced tetherin restricts vesicular stomatitis virus release in neurons. DNA Cell Biol 30, 965-974.

Schneider, W.M., Chevillotte, M.D., and Rice, C.M. (2014). Interferon-stimulated genes: a complex web of host defenses. Annu Rev Immunol 32, 513-545.

Soria, F.N., Pampliega, O., Bourdenx, M., Meissner, W.G., Bezard, E., and Dehay, B. (2017). Exosomes, an unmasked culprit in neurodegenerative diseases. Front Neurosci 11, 26.

Swiecki, M., Omattage, N.S., and Brett, T.J. (2013). BST-2/ tetherin: structural biology, viral antagonism, and immunobiology of a potent host antiviral factor. Mol Immunol 54, 132-139.

Swiecki, M., Scheaffer, S.M., Allaire, M., Fremont, D.H., Colonna, M., and Brett, T.J. (2011). Structural and biophysical analysis of BST-2/tetherin ectodomains reveals an evolutionary conserved design to inhibit virus release. J Biol Chem 286, 2987-2997.

Swiecki, M., Wang, Y., Gilfillan, S., Lenschow, D.J., and Colonna, M. (2012). Cutting edge: paradoxical roles of BST2/ tetherin in promoting type I IFN response and viral infection. J Immunol 188, 2488-2492.

Tai, Y.L., Chen, K.C., Hsieh, J.T., and Shen, T.L. (2018). Exosomes in cancer development and clinical applications. Cancer Sci 109, 2364-2374.

Tai, Y.T., Horton, H.M., Kong, S.Y., Pong, E., Chen, H., Cemerski, S., et al. (2012). Potent in vitro and in vivo activity of an Fc-engineered humanized anti-HM1.24 antibody against multiple myeloma via augmented effector function. Blood 119, 2074-2082.

Takeuchi, O., and Akira, S. (2010). Pattern recognition receptors and inflammation. Cell 140, 805-820.

Thurston, T.L., Ryzhakov, G., Bloor, S., von Muhlinen, N., and Randow, F. (2009). The TBK1 adaptor and autophagy receptor NDP52 restricts the proliferation of ubiquitin-coated bacteria. Nat Immunol 10, 1215-1221.

Tokarev, A., Skasko, M., Fitzpatrick, K., and Guatelli, J. (2009). Antiviral activity of the interferon-induced cellular protein BST-2/tetherin. AIDS Res Hum Retroviruses 25, 1197-1210.

Tokarev, A., Suarez, M., Kwan, W., Fitzpatrick, K., Singh, R., and Guatelli, J. (2013). Stimulation of NF-kappaB activity by the HIV restriction factor BST2. J Virol 87, 2046-2057.
Urata, S., and Kenyon, E. (2018). BST-2 controls T cell proliferation and exhaustion by shaping the early distribution of a persistent viral infection. PLoS Pathog 14, e1007172.

Van Damme, N., Goff, D., Katsura, C., Jorgenson, R.L., Mitchell, R., Johnson, M.C., et al. (2008). The interferoninduced protein BST-2 restricts HIV-1 release and is downregulated from the cell surface by the viral Vpu protein. Cell Host Microbe 3, 245-252.

Wainwright, D.A., Balyasnikova, I.V., Han, Y., and Lesniak, M.S. (2011). The expression of BST2 in human and experimental mouse brain tumors. Exp Mol Pathol 91, 440-446.

Wang, W., Nishioka, Y., Ozaki, S., Jalili, A., Abe, S., Kakiuchi, S., et al. (2009). HM1.24 (CD317) is a novel target against lung cancer for immunotherapy using anti-HM1.24 antibody. Cancer Immunol Immunother 58, 967-976.

Yokoyama, T., Enomoto, T., Serada, S., Morimoto, A., Matsuzaki, S., Ueda, Y., et al. (2013). Plasma membrane proteomics identifies bone marrow stromal antigen 2 as a potential therapeutic target in endometrial cancer. Int J Cancer 132, 472-484.

Address correspondence to:

Debasis Nayak, MVSc, PhD

Discipline of Biosciences and Biomedical Engineering Indian Institute of Technology Indore Simrol

Indore 453552

India

E-mail: nayakdn@iiti.ac.in

Juan C. de la Torre, PhD Department of Immunology and Microbial Science IMM-6

The Scripps Research Institute 10550 North Torrey Pines Road La Jolla, CA 92037

E-mail: juanct@scripps.edu

Dorian B. McGavern, PhD

National Institutes of Health Building 10, Room 5N240C, 10 Center Drive Bethesda, MD 20892

E-mail: mcgavernd@ninds.nih.gov

Received for publication April 3, 2019; received in revised form July 29, 2019; accepted July 29, 2019. 